\title{
Measurement of Cerebral Infarct Volume by Magnetic Resonance Imaging using $A B C / 2$ Method and its Correlation with Clinical Outcome using NIHSS Score
}

\author{
Veena Maradi ${ }^{1}$, Anand $\mathrm{K}^{2}$, Ashok Kumar ${ }^{3}$ \\ ${ }^{1}$ Assistant Professor, Department of Radio-Diagnosis, Karnataka Institute of Medical Sciences, Hubballi, Karnataka, ${ }^{2}$ Associate Professor, \\ Department of Radio-Diagnosis, M.S.Ramaiah Medical College, Bengaluru, Karnataka, ${ }^{3}$ Professor and Head, Department of Radio- \\ Diagnosis, M.S.Ramaiah Medical College, Bengaluru, Karnataka, India
}

Corresponding author: Dr Anand K, Associate Professor, Department of Radio-Diagnosis, M.S.Ramaiah Medical College, Bengaluru, Karnataka, India

DOI: http://dx.doi.org/10.21276/ijcmsr.2019.4.2.32

How to cite this article: Veena Maradi, Anand K, Ashok Kumar. Measurement of cerebral infarct volume by magnetic resonance imaging using $\mathrm{ABC} / 2$ method and its correlation with clinical outcome using NIHSS score. International Journal of Contemporary Medicine Surgery and Radiology. 2019;4(2):B144-B149.

\section{A B S T R A C T}

Introduction: Stroke is a second leading cause of death globally and is a major cause of long term disability. Study aimed to measure the cerebral infarct volume by MRI using $A B C / 2$ method and its correlation with clinical outcome using NIHSS score.

Material and Methods: This was a prospective study done over a period of one and half years. About 60 adult cases of acute ischemic stroke, who underwent MR imaging (DWI sequence) in department of Radio-Diagnosis, M.S.Ramaiah Medical College, Bengaluru, were included in the study. The cerebral infarct volume caw calculated on MRI study. The National Institute of Health Stroke Severity score was done in all the cases. The correlation between cerebral infarct volume and NIHSS was seen at the end of 90 days for clinical outcome. The current study evaluates the relationship between NIHSS score and infarct volume and how it can be used to assess the outcome of the neurological severity ninety days after acute stroke.

Results: Out of 60 cases, clinical outcome was good in 41 cases with an infarct volume between 10-39 cc, poor in 13 cases with an infarct volume between $40-89 \mathrm{cc}$ and 6 patients died with an infarct volume above $100 \mathrm{cc}$. Poor outcome was seen in cases with NIHSS score above 25.

Conclusion: The infarct volume between 10-40 cc showed good prognosis, infarct volume between 40 and 89 cc showed poor prognosis while volume above 100 cc resulted in $100 \%$ mortality. As the infarct volume increases the NIHSS score also increases on follow up study predicting the clinical outcome with the infarct volume estimated at the onset of stroke. From this study we conclude that the acute stroke volume can be used to predict the stroke severity by using NIHSS score.

Keywords: Cerebral Infarct Volume, DWI-Magnetic Resonance Imaging, ABC/2 Method, NIHSS Score

\section{INTRODUCTION}

Hippocrates, the father of medicine, first recognized stroke over 2,400 years ago. At this time stroke was called apoplexy, which means "struck down by violence" in Greek. This was due to the fact that a person developed sudden paralysis and change in well-being. ${ }^{1}$ The two major categories of stroke are ischemic (lack of blood and hence oxygen to an area of the brain) and haemorrhagic (bleeding from a burst or leaking blood vessel in the brain) stroke. ${ }^{2}$ Cerebrovascular ischemia from thromboembolism or atherosclerotic stenosis leads to acute infarct with or without hemorrhage.

MRI provides critical information in the setting of acute stroke that can be used to confirm a diagnosis and direct both acute therapeutic interventions and long-term treatment decisions. MRI can identify regions of infarct and vessel occlusion. With appropriate processing, MRI has the potential to identify regions of core infarct (ischemic core) and salvageable tissue (penumbra). ${ }^{3}$ MRI is sensitive to detect acute infarct using diffusion weighted sequence. The ability to detect early infarct in patients with acute stroke has made DWI a powerful technique in the evaluation of stroke patients. $^{3}$ NIHSS score is a valid reproducible scale that measures major neurological deficit. However this is a clinical assessment. The current study evaluates the relationship of acute infarct volume after the onset of stroke and NIHSS score with the outcome at the end of 90 days. Study aimed to measure the cerebral infarct volume by MRI using $\mathrm{ABC} / 2$ and its correlation with clinical outcome using NIHSS score.

\section{MATERIAL AND METHODS}

The present study was a prospective hospital based study done over a period of one and half years from November 2012 to April 2014. The study was done in the department 
of Radio-diagnosis, M.S. Ramaiah Medical College and Hospital, Bengaluru, India. About 60 cases of acute ischemic stroke who underwent MR imaging were included in the study. Acute stroke volume was assessed in Diffusion weighted Image (DWI) sequence. MRI lesions for acute or subacute volumes were measured on DWI images and the infarct volumes were calculated using Ellipsoid method ${ }^{4}-$ $\mathrm{ABC} / 2$ method, where $\mathrm{A}$ is the longest dimension in $\mathrm{X}$ axis, $\mathrm{B}$ is longest perpendicular dimension to $\mathrm{X}$ axis $(\mathrm{Y})$, and $\mathrm{C}$ is total length in $Z$ dimension.

In all cases (figure 1,2,3), the clinical details such as age and gender of the patient, clinical history, indication for MRI, risk factors for the cerebral infarct were noted.

The DWI MRI was done within 24 hours for all the cases and a baseline NIHSS score was taken on the day of admission itself. The NIHSS score was repeated at the end of three months (90 days) for all the cases so as to predict the stroke severity and clinical outcome of the patient which was assessed at the end of 90 days.

NIHSS: The National Institute of Health Stroke Scale is a valid, reproducible scale that measures neurological deficit. It assesses the extent of neurological deficit after an episode of stroke. $^{5}$ There is a total of 42 points. There are 11 parts in the NIHSSS, with 13 specific tests being performed. It examines the level of consciousness, vision and gaze, facial palsy and extremity weakness, limb ataxia, sensory loss, language and dysarthria, and neglect. A patient with a completely normal neurological exam and normal mental status will have NIHSS score of 0 . The maximum recordable score is 42 . However, since acute ischemic stroke causes unilateral paralysis, the maximum score is actually 31 for stroke patients with complete hemiparesis, hemianopia and aphasia. Patients with an NIHSS score greater than $15-20$ are considered to have severe stroke clinically. ${ }^{6}$

\section{NIHSS score and Patient Outcome}

- Total scores range from 0 - 42 with higher values representing more severe infarcts.

- $\quad>25$ Very severe neurological impairment

- 15-24 Severe impairment

- 5-14 Moderately severe impairment

- $\quad$ 5 Mild impairment

- A 3-point (or greater) increase on the NIHSS administered serially indicates progression of stroke.

\section{Inclusion criteria}

Adult patients detected to have an acute infarct on MRI study.

\section{Exclusion criteria}

Patients less than 16 years, patients detected to have lacunar and/or old infarcts, patients with cerebral hemorrhage and patients with cerebral mass lesions, patients with previous neurological deficit were excluded from the study.

\section{STATISTICAL ANALYSIS}

Quantitative variables like age, infarct volume were expressed in terms of descriptive statistics like mean and standard deviation. The entire qualitative variables were expressed in terms of proportion like Pearson's correlation.

\section{RESULTS}

A total of 60 adult patients were studied.

Categorization of patients based on their gender: There were $36(60 \%)$ male and $24(40 \%)$ female patients and the male to female ratio was $1.5: 1$.

In this study the peak incidence of neurological deficits in males occurred in the age group between $60-69$ years that is 13 patients (21.4\%). In females, the peak incidence occurred in the age group between $70-79$ years that is 09 patients (15\%) (table-1).

Number of hypertensive patients in the study subjects: In this study out of the 60 patients, 21 (35\%) patients had hypertension and 39 patients had no history of hypertension

\begin{tabular}{|l|c|c|c|}
\hline \multirow{2}{*}{ Age in years } & \multicolumn{2}{|c|}{ Gender } & \multirow{2}{*}{ Total } \\
\cline { 2 - 3 } & Female $\mathbf{n}(\%)$ & Male $\mathbf{n}(\%)$ & \\
\hline $40-49$ & $02(3.3)$ & $03(05)$ & $05(4.0)$ \\
\hline $50-59$ & $06(10)$ & $09(15)$ & $15(12.0)$ \\
\hline $60-69$ & $07(12)$ & $13(21.4)$ & $20(28.0)$ \\
\hline $70-79$ & $09(15)$ & $11(18.3)$ & $20(15.8)$ \\
\hline Total & 24 & 36 & 60 \\
\hline & $40.3 \%$ & $59.7 \%$ & $100 \%$ \\
\hline
\end{tabular}

Table-1: Distribution of age and gender of the patients

\begin{tabular}{|l|c|c|}
\hline $\begin{array}{l}\text { Both Hypertension and } \\
\text { Diabetes Mellitus }\end{array}$ & Number (n) & Percentage (\%) \\
\hline Absent & 38 & $63.3 \%$ \\
\hline Present & 22 & $36.7 \%$ \\
\hline Total & 60 & $100 \%$ \\
\hline Table-2: Distribution of patients with both hypertension and \\
diabetes mellitus \\
\hline
\end{tabular}

\begin{tabular}{|l|c|c|}
\hline $\begin{array}{l}\text { Cerebral infarct } \\
\text { volume (in cc) }\end{array}$ & $\begin{array}{c}\text { No. of patients } \\
(\mathbf{n = 6 0})\end{array}$ & Percentage (\%) \\
\hline $10-39$ & 41 & $68 \%$ \\
\hline $40-69$ & 10 & $16 \%$ \\
\hline $70-99$ & 03 & $05 \%$ \\
\hline $100-140$ & 06 & $10 \%$ \\
\hline \multicolumn{2}{|c|}{ Table-3: Volume of the cerebral infarcts } \\
\hline
\end{tabular}

\begin{tabular}{|l|c|c|}
\hline $\begin{array}{l}\text { Number of cases } \\
(\mathbf{n}=\mathbf{6 0})\end{array}$ & $\begin{array}{c}\text { Cerebral infarct } \\
\text { volume (in cc) }\end{array}$ & Clinical prognosis \\
\hline 41 & $10-39$ & Good \\
\hline 13 & $40-99$ & Poor \\
\hline 6 & $>100$ & Mortality \\
\hline \multicolumn{2}{|c|}{ Table-4: Volume of cerebral infarct and clinical prognosis } \\
\hline
\end{tabular}

\begin{tabular}{|l|c|c|}
\hline NIHSS Score & No. of patients & Percentage (\%) \\
\hline$<5$ & 28 & $46.7 \%$ \\
\hline $5-14$ & 12 & $20 \%$ \\
\hline $15-24$ & 9 & $15 \%$ \\
\hline$>25$ & 11 & $18.3 \%$ \\
\hline Total & 60 & $100 \%$ \\
\hline \multicolumn{3}{|c|}{ Table-5: NIHSS Score in this study } \\
\hline
\end{tabular}



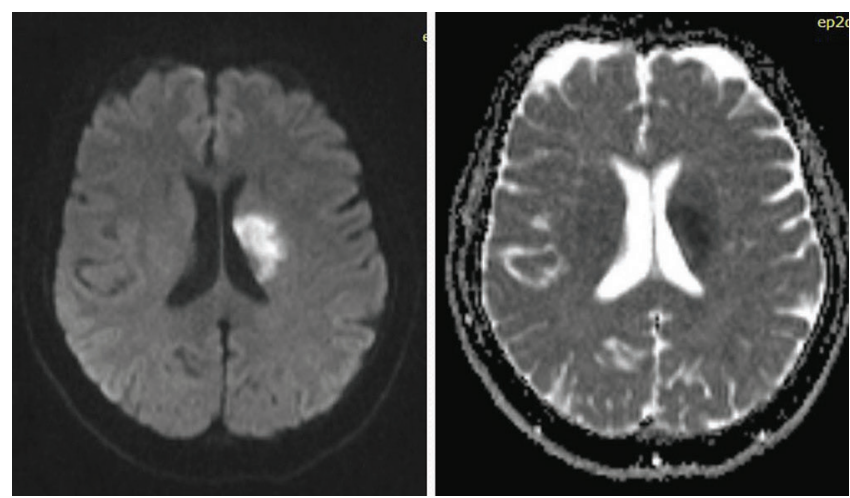

Figure-1: A 50 years old female (Hosp. No: 366042) presented with sudden onset of altered sensorium. Axial images show hyperintensity in DWI and hypointensity in ADC in the left corona radiata suggestive of acute cerebral infarct with an infarct volume of $12 \mathrm{cc}$ and NIHSS score of 3

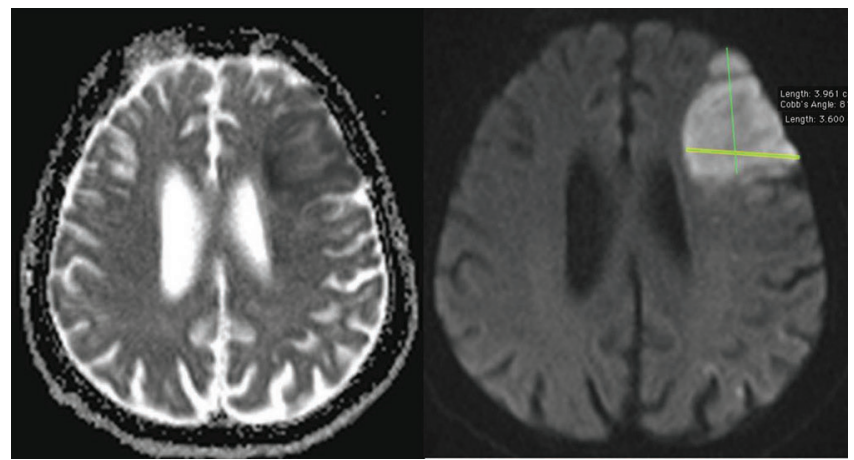

Figure-2: MR images of 70 years female (Hosp. No: 358886) presented with sudden onset of slurring of speech, deviation of angle of mouth to the left side and weakness of right upper limb and lower limb, showing area of diffusion restriction in the fronto- parietal lobe suggestive of acute infarct with an infarct volume of $34 \mathrm{cc}$ and NIHSS score of 12. However, patient's speech and right hemiparesis improved.
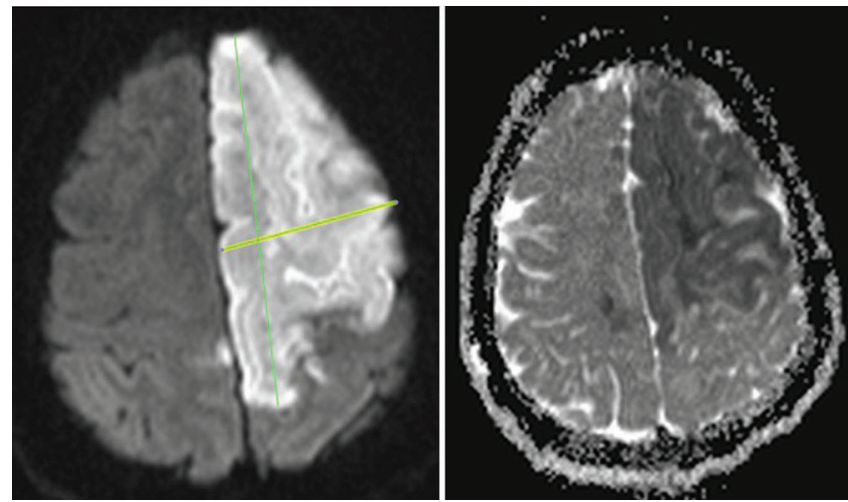

Figure-3: A 46 years female (Hosp. No - 339749) presented with weakness in right upper and lower limbs and loss of speech. A. Axial DWI b 100 sequence shows hyperintense signal in the left frontal lobe. B. Axial ADC shows hypointensity suggestive of acute infarct. The cerebral infarct volume was $90 \mathrm{cc}$ and NIHSS score was 24. However, this patient died.

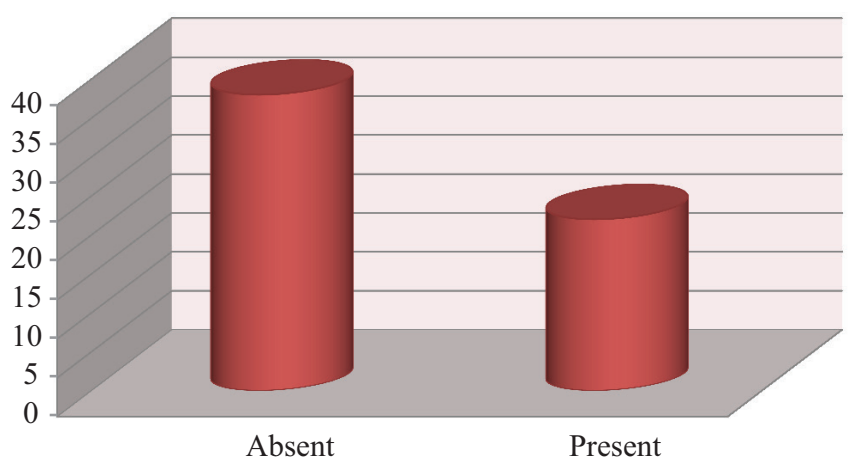

Patients with both diabetes mellitus and hypertension

Figure-4: Distribution of patients with both hypertension and diabetes mellitus

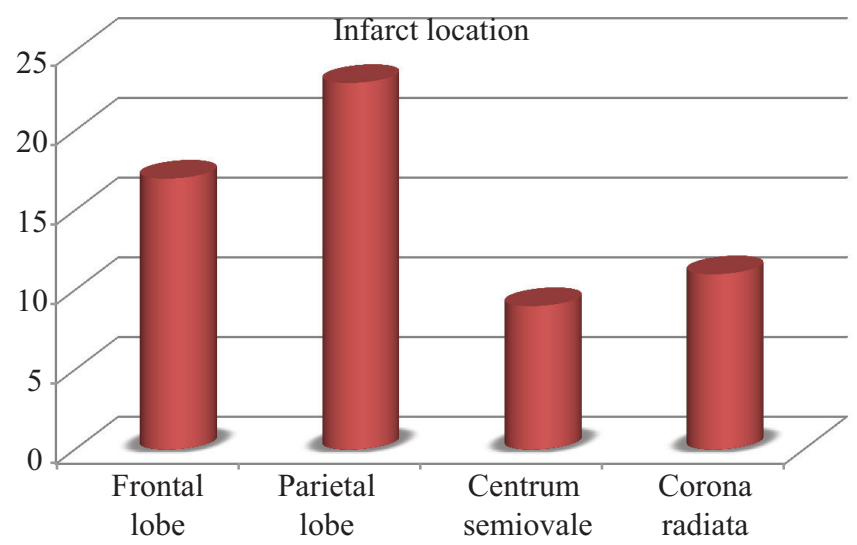

Figure-5: Location of the infarct

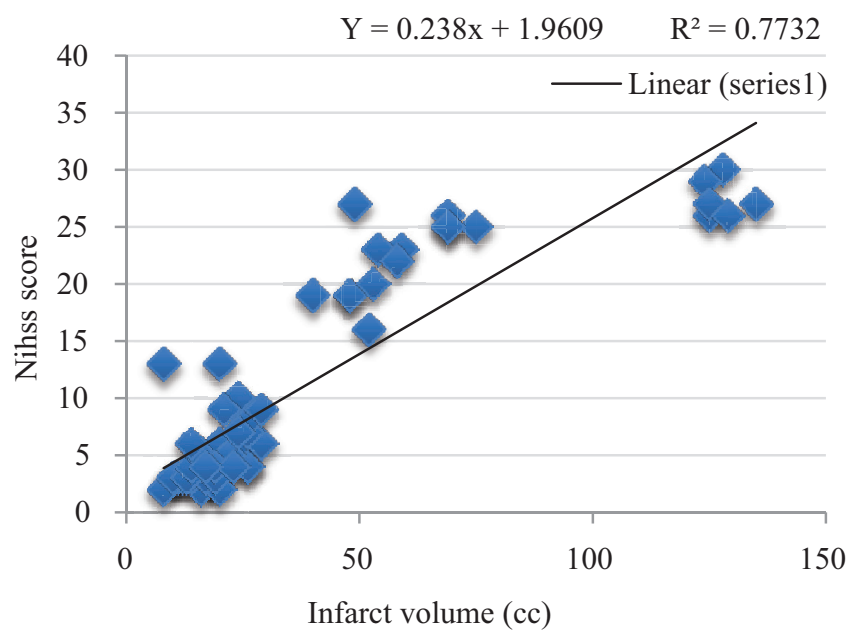

Figure-6: Correlation of infarct volume and NIHSS score

(table-2).

In this study out of the 60 patients, $22(36.7 \%)$ patients had both hypertension and diabetes mellitus and 38 (63.3\%) patients had no history of either hypertension or diabetes mellitus (figure-4). Infarcts in parietal lobe were most common (figure-5).

In this study out of the 60 patients, $41(68 \%)$ patients had infarct volume between 10-39 cc, 10 (16) patients had infarct volume between 40-69 cc, 03 (05\%) patients between $70-99$ cc and 06 (10\%) patients had infarct volume between 100$140 \mathrm{cc}$ (table-3).

Of 60 cases, clinical outcome was good in 41 cases with an 
infarct volume between $10-39 \mathrm{cc}$, poor in 13 cases with an infarct volume between $40-89 \mathrm{cc}$ and 6 patients died with an infarct volume above $100 \mathrm{cc}$ (table-4). In this study, out of the 60 patients, $28(46.7 \%)$ patients had NIHSS score less than 5 (table-5).

Pearson's correlation was used to correlate between cerebral infarct volume and NIHSS score with a correlation coefficient of $\mathrm{r}=0.87: \mathrm{P}<0.001$ which was statistically significant. This defines a linear association. It showed the regression t-test as $\mathrm{t}=-5.550$ with $95 \%$ confidence interval $-1.024-2.172$ and regression correlation coefficient of $r 2=0.300 ; p<0.001$. Hence, infarct volume proved to be a good predictor of NIHSS score on follow up study. As the NIHSS score increases the neurological deficit becomes more severe showing a positive correlation between NIHSS and infarct volume (figure-6).

\section{DISCUSSION}

MRI Diffusion Weighted Imaging (DWI) is more than 95\% sensitive when detecting infarcts within the first 6 hours from the onset of symptoms and is currently the most reliable method for infarct identification. ${ }^{7}$ DWI detects brain infarcts within the first hour of onset of symptoms and it also allows differentiation of acute and chronic lesions. DWI can also reveal multiple acute brain infarcts which in turn guides to look for specific etiology and also to plan preventive strategies for patients presenting with lacunar strokes. ${ }^{8} \mathrm{MR}$ images produced with this technique depend on the motion of water molecules in the brain. DWI enhancement appears within 5- 10 minutes after the onset of a stroke and lasts for up to two weeks. This is a major breakthrough when compared to CT, since the latter can only detect acute infarct changes after 4-6 hours. CT is therefore used to rule out hemorrhagic stroke which is contraindication for the use of tissue plasminogen activator. ${ }^{9}$ When compared to CT, DWI is $33 \%$ more sensitive in the detection of middle cerebral artery involvement when identifying acute infarctions. Diffusion weighted imaging is extremely valuable due to its ability to reliably detect hyper acute ischaemic infarcts when other imaging modalities are still normal. It also assists with determining the age of the infarct and to distinguish between small lacunar infarcts and chronic micro-vascular changes. Diffusion weighted imaging (DWI) MRI study is considered as the gold standard imaging modality in the diagnosis of acute ischemic stroke. It is not widely available and has the drawback of taking more time to perform the test which is of concern, especially in the acute therapeutic window. ${ }^{10}$

While CT imaging is more readily available and less expensive than magnetic resonance imaging (MRI), it is of limited use in predicting infarct volume in the acute setting. ${ }^{10}$ DWI in MRI is considered to have greater sensitivity than conventional MRI and computed tomography scan (CT scan) in identifying acute ischaemic lesions. ${ }^{11}$

MRI protocols such as DWI stroke volume, infarct core volume, and DWI ASPECTS system that measure the infarct volume in acute ischemic stroke have shown high inter-reader reliability and good prediction power for clinical outcome. ${ }^{12}$

In the present study the $\mathrm{ABC} / 2$ method was used for estimation of stroke volume. This geometric method gives rapid and accurate clinical estimation of stroke volume. It requires less than a minute to perform and has good intrarater and inter-rater reliability. This is a simple, accurate and quick method to measure infarct volume. Application of $\mathrm{ABC} / 2$ also allows for accurate stroke volume estimation in intensive care units for discussion of prognosis and planning interventions. The ease of applying $\mathrm{ABC} / 2$ method in the acute stroke setting provides a guide for therapeutic choices. ${ }^{4}$ NIHSS was designed in 1989, which includes 15 items. It is a neurological examination scale for the evaluation of consciousness, eyeball movements, visual field, limb motor and sensations, limb coordination, speech, cognition and attention in the form of grade. The total score is 0 to 42 points. Many studies have proved that the NIHSS score is reliable and has good validity. ${ }^{13}$

In the present study, hypertension and diabetes were seen in 22 of $60(36.7 \%)$ cases.

Elevated blood pressure or hypertension is a known risk factor for ischemic stroke and intracerebral hemorrhage. ${ }^{14}$ It is a direct, continuous and independent risk factor for stroke. ${ }^{14}$ Elevated blood pressures along with type 2 diabetes mellitus increase the risk of stroke. ${ }^{15}$ Tight control of blood pressure and maintaining it around $<130 / 80 \mathrm{~mm} \mathrm{Hg}$ is recommended for hypertensive diabetic patients with an increased primary stroke risk. ${ }^{16}$

Location of the cerebral infarct: Ischemic strokes in anterior circulation ie due to thrombi or emboli from the carotid circulation are more common accounting for approximately $70 \%$ of all cases and around $20 \%$ strokes arise in posterior circulation territory. ${ }^{17}$ In our study also the frontal lobe and parietal lobes were the common sites for infarcts accounting for 40/60 (66\%) cases.

Volume of the cerebral infarcts and NIHSS correlation: In the present study, $68 \%$ patients had infarct volume of 10 to 39 cc.

The studies done by Tong et al and Lovblad et al shown a correlation between the NIHSS and infarct volume in patients with acute ischemic stroke. ${ }^{18,19}$

While some studies show that infarct volume does not add to the NIHSS score obtained on day 5 in predicting 3-month outcome, ${ }^{20}$ other studies suggested that infarct volume adds to the baseline NIHSS score in predicting outcome. ${ }^{21-23}$ In our study a baseline NIHSS was taken and outcome was assessed at the end of three months. In addition, in case series with a significant proportion of mild strokes, there is a strong association between infarct growth and 3-month functional outcome, ${ }^{24}$ In our study it was observed that higher the infarct volume, more was the NIHSS score and poorer was the clinical outcome.

So with NIHSS as the score increases, the neurological deficit becomes more severe. This can explain the positive correlation one gets for NIHSS versus infarct volume, thus they are directly proportional as the infarct volume increases so does the neurological deficit.

Though there is a correlation between infarct volume and stroke severity, the degree of severity does not always correlate with infarct volume. For example, even though 
some subcortical infarcts are small in size, they may produce a significant clinical deficit. ${ }^{25}$

Yaghi et $\mathrm{al}^{26}$ in their study attempted to determine the association between DWI lesion(s) volume and the total NIHSS score and also NIHSS component scores in patients with minor stroke. They observed that patients with mild stroke symptoms usually had relatively small infarcts on neuroimaging.

Patients with an NIHSS score greater than $15-20$ are considered to have severe stroke clinically. This study showed as NIHSS score increases the neurological deficit becomes more severe. There is a positive correlation between NIHSS score and infarct volume. They are directly proportional and as the infarct volume increases so does the neurological deficit. Our findings are similar to the study done by Young et al. ${ }^{27}$

\section{CONCLUSION}

This study is unique in establishing the relationship among the infarct volume and NIHSS score and the prediction of clinical outcome after 90 days. The infarct volume between 10-40 cc showed good prognosis, infarct volume between 40 and $89 \mathrm{cc}$ showed poor prognosis while volume above $100 \mathrm{cc}$ resulted in 100\% mortality. As the infarct volume increases the NIHSS score also increases on follow up study predicting the clinical outcome with the infarct volume estimated at the onset of stroke. From this study we conclude that the acute stroke volume can be used to predict the stroke severity by using NIHSS score.

\section{REFERENCES}

1. Gund BM, Jagtap PN, Ingale VB, Patil RY. Stroke: A Brain Attack. IOSR Journal of Pharmacy 2013;3(8):0123.

2. Lees KR, Zivin JA, Ashwood T, Davalos A, Davis SM, Diener HC, et al. NXY-059 for acute ischemic stroke. N Engl J Med. 2006.

3. Dhamija RK, Donnan GA. The role of neuroimaging in acute stroke. Ann Indian Acad Neurol 2008;11, Suppl S1:12-23

4. Sims JR, Gharai LR, Schaefer PW, Vangel M, Rosenthal ES, Lev $\mathrm{MH}$, et al. ABC/2 for rapid clinical estimate of infarct, perfusion, and mismatch Neurology 2009;72(24):2104-10.

5. Saver JL, Johnston KC, Homer D, Wityk R, Koroshetz W, Truskowski LL, et al. Infarct volume as a surrogate or auxiliary outcome measure in ischemic stroke clinical trials. The RANTTAS investigators. Stroke 1999;30(2):293-298.

6. Yuh W, Crain MR, Loes DJ. MR imaging of cerebral ischemia: Findings in the first 24 hours. Am J Neuroradiol 1991; 12(5):621-629.

7. Moseley M, Kucharczyk J, Mintorovitch J. Diffusionweighted MR imaging of acute stroke: correlation with T2-weighted and magnetic susceptibility-enhanced MR imaging in cats. Am J Neuroradiol 1990; 11(4):423-429.

8. Appelros P, Te'rent A. Characteristics of the National Institute of Health Stroke Scale: results from a population-based stroke cohort at baseline and after one year. Cerebrovasc Dis. 2004;17(1):21-27.
9. Wardlaw JM, Keir SL, Bastin ME, Armitage PA, Rana AK. Is diffusion imaging appearance an independent predictor of outcome after ischemic stroke? Neurology. 2002;59(6):1381-7.

10. Merino JG, Warach S. Imaging of acute stroke. Nat Rev Neurol. 2010; 6(1):560-71

11. Barber PA, Darby DG, Desmond PM, et al. Identification of major ischemic change. Diffusionweighted imaging versus computed tomography. Stroke 1999;30(5):2059-65

12. Han M, Choi JW, Rim NJ, Kim SY, Suh, H-II, Lee KS, et al. Cerebral infarct volume measurements to improve patient selection for endovascular treatment.

13. Kwiatkowski TG, Libman RB, Frankel M, et al. Effects of tissue plasminogen activator for acute ischemic stroke at one year. National Institute of Neurological Disorders and Stroke Recombinant Tissue Plasminogen Activator Stroke Study Group. N Engl J Med 1999;340(1):17817.

14. Straus SE, Majumdar SR, McAlister FA. New evidence for stroke prevention. JAMA 2002;288(2):1388-1395.

15. Sacco RL. Reducing the risk of stroke in diabetes: what have we learned that is new?Diabetes Obes Metab. 2002;4 (suppl 1):S27-S34.

16. Position Statement.Hypertension management in adults with diabetes. Diabetes Care.2004;27(1):S65-S67.

17. Baird A. (2013). Anterior Circulation Stroke. eMedicine. Retrieved from http://emedicine.medscape. com/article/1159900-overview\#aw2aab6b2.

18. Tong DC, Yenari MA, Albers GW, O’Brien M, Marks MP, Moseley ME. Correlation of perfusionand diffusion-weighted MRI with NIHSS score in acute ( $<6.5$ hour) ischemic stroke. Neurology 1998; 50(5):864-70.

19. Lovblad KO, Baird AE, Schlaug G, Benfield A, Siewert $\mathrm{B}$, Voetsch B, et al. Ischemic lesion volumes in acute stroke by diffusion-weighted magnetic resonance imaging correlate with clinical outcome. Ann Neurol 1997;42(3):164-70.

20. Johnston KC, Barrett KM, Ding YH, Wagner DP. Clinical and imaging data at 5 days as a surrogate for 90-day outcome in ischemic stroke. Stroke 2009;40(1):1332-3.

21. Campbell BC, Tu HT, Christensen S, Desmond PM, Levi CR, Bladin CF, et al. Assessing response to stroke thrombolysis: validation of 24-hour multimodal magnetic resonance imaging. Arch Neurol 2012; 69(5):46-50.

22. Vogt G, Laage $R$, Shuaib A, Schneider A. Initial lesion volume is an independent predictor of clinical stroke outcome at day 90: an analysis of the Virtual International Stroke Trials Archive (VISTA) database. Stroke 2012;43(1):1266-72.

23. Vagal AS, Sucharew H, Prabhakaran S, Khatri P, Jovin $\mathrm{T}$, Michel $\mathrm{P}$, et al. Final infarct volume discriminates outcome in mild strokes. Neuroradiol J. 2015;28(4):4048.

24. Khatri P, Conaway MR, Johnston KC. Ninetyday outcome rates of a prospective cohort of consecutive patients with mild ischemic stroke. Stroke 2012;43(1):560-2.

25. Duan Z, Fu C, Chen B, Xu G, Tao L, Tang T, et al. 
Lesion patterns of single small subcortical infarct and its association with early neurological deterioration. Neurol Sci 2015; 36(8):1851-7.

26. Yaghi S, Herber C, Boehme AK, Andrews H, Willey ZJ, Rostanski SK. The Association between Diffusion MRIDefined Infarct Volume and NIHSS Score in Patients with Minor Acute Stroke: Diffusion MRI-Defined Infarct Volume. J Neuroimaging. 2017;27(4):388-391.

27. Young FB, Weir CJ, Lees KR. Comparison of the National Institutes of Health Stroke Scale with disability outcome measures in acute stroke trials. Stroke 2005;36(1):2187-92.

Source of Support: Nil; Conflict of Interest: None

Submitted: 17-04-2019; Accepted: 11-05-2019; Published online: 20-06-2019 